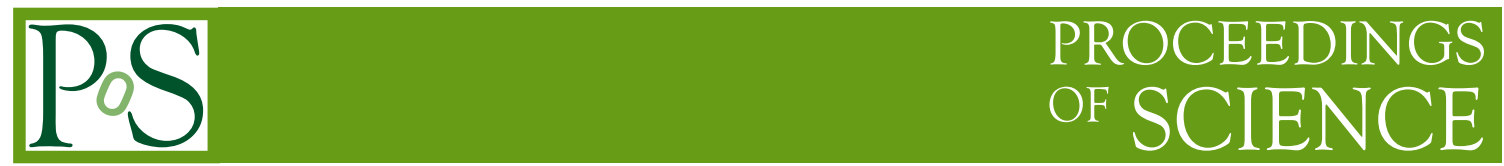

\title{
Recent results on charmonium transition at BESIII
}

\section{Gang LI*i}

Institute of High Energy of Physics, Beijing, P.R. China

E-mail: LI.Gang@mail.ihep.ac.cn

Recent results of the transitions between Charmonium spectrum at Beijing Spectrometers III (BES-III) experiment are presented. This report includes the following topics: the first measurements of the transition rates of $\psi^{\prime} \rightarrow \pi^{0} h_{c}$ and $h_{c} \rightarrow \gamma \eta_{c}$, width of $h_{c}$, and improved measurement of the mass of the $h_{c}$; Also and the first observation of $\psi^{\prime} \rightarrow \gamma \gamma J / \psi$ two-photon transition. All these measurements are based on the world largest $\psi^{\prime}$ data sample collected with the BES-III detector at the Beijing Electron Positron Collider II (BEPC-II).

35th International Conference of High Energy Physics - ICHEP2010,

July 22-28, 2010

Paris France

*Speaker.

${ }^{\dagger}$ On behalf of BESIII Collaboration. 


\section{Introduction}

The standard model (SM) has been widely accepted as the fundamental theory of elementary particle physics according to its successful description of the experimental phenomena. However, SM still leave many unanswered questions. These questions may be classified into two categories: the first is relevant to very high energy region including such as search for Higgs particles and new physics beyond SM; the second is for the non-perturbative physics at long-distance, is mostly related to the Quantum Chromodynamics (QCD).

Operating at $2 \sim 4.6 \mathrm{GeV}$ energy region, BES(BES-I and BES-II)/BEPC aimed to contribute to the non-perturbative physics, because it spanned the energy range where both short-distance and long-distance effects can be probed. Eventually it has produced fruitful physics results such as the $\tau$ mass measurement, $R$-value measurement, new resonances and enhancement observation, and non$D \bar{D}$ decay of $\psi(3770)$, etc. However, higher precision measurement and a better understanding of rare processes need larger statistics, that's why BEPC is updated to BEPC-II since 2004. The updated machine is a high luminosity double-ring collider and still works at the charm energy region. Correspondingly, BES-II is updated to BES-III to handle the upcoming huge data and provide much more precise measurement based on enhanced sensitivities and resolutions in each sub-detector as well[1].

Since the first $e^{+} e^{-}$collision event in BES-III, BES-III has already collected about $106 \mathrm{M}$ $\psi(2 S), 226 \mathrm{M} J / \psi$ and $1 \mathrm{fb}^{-1} \psi(3770)$ events till now. In which each of the $\psi(2 S)$ and $J / \psi$ sample is the largest one in the world. With these data samples and that in future, BES collaboration will contribute a lot to the physics of $\tau$-charm region. There will be abundant and interesting physics such as light hadron spectroscopy, charmonium states, new states above open charm threshold $(\mathrm{X}, \mathrm{Y}, \mathrm{Z}), J / \psi$ and $\psi(2 S)$ hadronic decays, electromagnetic form factors and QCD cross section (R value) and high precision $\tau$ mass and charm physics near threshold, open charm physics and search for new physics. In this report we only focus on the two recent charmonium results at BES-III: the measurement of $h_{c}$ via $\psi(2 S) \rightarrow \pi^{0} h_{c}$, the observation of branch fraction of two-photon transition of $\psi^{\prime} \rightarrow \gamma \gamma J / \psi$

\section{Recent Charmonium Transitions}

\subsection{Observation of $h_{c}$}

$h_{c}$ is a ${ }^{1} P_{1}$ charmonium state $(c \bar{c})$, which is predicted for a long time by theoretical physics based on simple quark model. And because it is expected as a charmonium singlet state, there is non-strong spin-spin interaction and its mass would be the average of masses of triple 1P-wave states, i.e. $\chi_{c 0,1,2}$. Till now, it only be found via $\psi(2 S) \rightarrow \pi^{0} h_{c}$, and only CLEOc[2] gives its mass and product branching fractions before BES-III results.

We apply two methods to measure the mass and width of $h_{c}$ and the transition rate of $\psi(2 S) \rightarrow$ $\pi^{0} h_{c}$ : one is an E1-tagged analysis of $\psi(2 S) \rightarrow \pi^{0} h_{c}, h_{c} \rightarrow \gamma \eta_{c}$ in witch the radiative photon from $h_{c}$ decay is tagged; another one is the inclusive analysis of $\psi(2 S) \rightarrow \pi^{0} h_{c}$. Here about $106 \mathrm{M}$ $\psi(2 S)$ events collected by BES-III at BEPC-II in March and April of year 2009 has been used. Notice in these two methods the performance of BES-III electromagnetic calorimeter (EMC) is crucial because it is the key detector to measure the energy and direction of photons from $\pi^{0}$ or 
$h_{c}$ radiative transitions. In BES-III EMC, energy resolution is good to achieve $2.5 \%$ at $1 \mathrm{GeV}$. Further effects, such as using time information to reject beam gas and add energy deposit in TOF to improve efficiency, have been applied to improve detection and resolution also. From Fig.1, the comparison between Monte Carlo (MC) and data shows good coincidence.

Fig.2(a) shows the $\pi^{0}$ recoil mass from E1-tagged process $\psi(2 S) \rightarrow \pi^{0} h_{c}, h_{c} \rightarrow \gamma \eta_{c}$, which is fitted by the signal convolved with the resolution and plus backgrounds. Here the signal of $h_{c}$ is described by non-relativistic Breit-Wiger, the resolution is obtained from MC simulation and parameterized by double-Gaussian, and backgrounds are represented by the $\pi^{0}$ recoil mass spectrum in the sideband of the E1 photon. The mass and width of $h_{c}$ and normalization floated when fit. Obtained results $M\left(h_{c}\right)=3535.40 \pm 0.13 \mathrm{MeV}, N\left(h_{c}\right)=3679 \pm 319$ and $\Gamma\left(h_{c}\right)=0.73 \pm$ $0.45 \mathrm{MeV}$ are consistent with CLEOc's result[2]: $M\left(h_{c}\right)=3525.35 \pm 0.23 \mathrm{MeV}$ and theoretical predictions. Notice the width of $h_{c}$ is the first observation and only statistical errors are presented here.

Fig.2(b) shows the $\pi^{0}$ recoil mass from inclusive $\psi^{\prime} \rightarrow \pi^{0} h_{c}$, which is fitted by the signal convolved with the resolution and plus backgrounds, and in this fit the mass and width of $h_{c}$ are fixed to the values obtained from previous E1-tagged analysis. Backgrounds are parameterized by a 4th-order polynomial. Combined with the previous E1-tagged results, we can get the branch fraction $\operatorname{Br}\left(\psi^{\prime} \rightarrow \pi^{0} h_{c}\right)=(8.4 \pm 1.3) \times 10^{-4}$ and $\operatorname{Br}\left(h_{c} \rightarrow \gamma \eta_{c}\right)=(54.3 \pm 6.7) \%$, they are both first observation in the world. And these results are also consistent with CLEOc's product branch fractions results[2]: $\operatorname{Br}\left(\psi^{\prime} \rightarrow \pi^{0} h_{c}\right) \times \operatorname{Br}\left(h_{c} \rightarrow \gamma \eta_{c}\right)=(4.22 \pm 0.44 \pm 0.52)^{-4}$.

This work has already been published in Ref. [3].
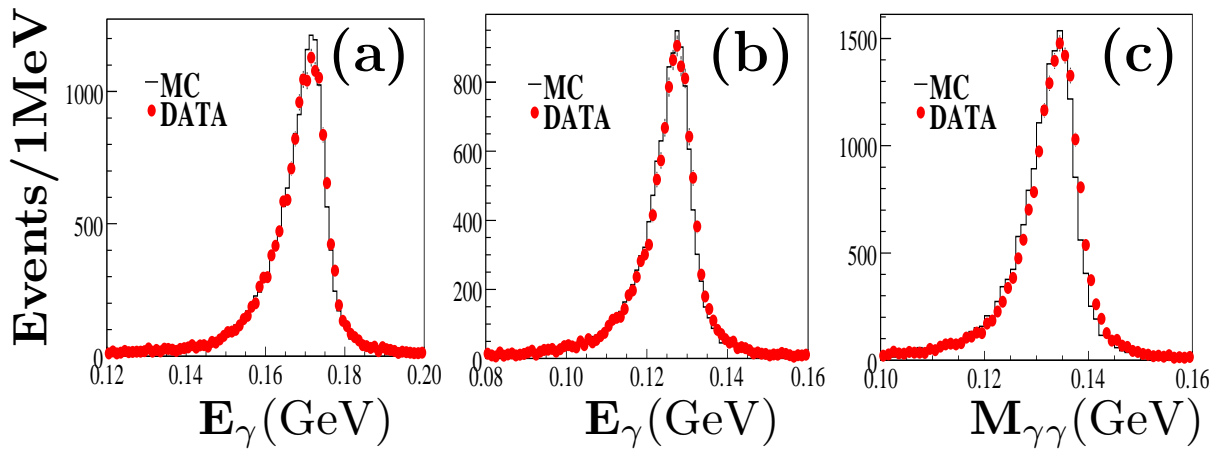

Figure 1: Comparisons between MC (lines) and data (dots): (a) energy distribution of the radiative photon in $\psi^{\prime} \rightarrow \gamma \chi_{c 1}, \chi_{c 1} \rightarrow \gamma J / \psi, J / \psi \rightarrow l^{+} l^{-}$; (b) energy distribution of the radiative photon in $\psi^{\prime} \rightarrow \gamma \chi_{c 2}, \chi_{c 2} \rightarrow$ $\gamma J / \psi, J / \psi \rightarrow l^{+} l^{-} ;$(c) invariant mass distribution of $\pi^{0}$ in $\psi^{\prime} \rightarrow \pi^{0} \pi^{0} J / \psi, J / \psi \rightarrow l^{+} l^{-}$;

\subsection{Two-photon transition of $\psi(2 S)\left(\psi^{\prime}\right) \rightarrow \gamma \gamma J / \psi$}

The two-photon spectroscopy has been always a very powerful tool for the study of the excitation level of different size-scale systems, such as molecules, atomic hydrogen and positronium $[4,5,6]$. This transition in hydrogen is specially useful in the study of cosmological hydrogen recombination in the universe [7]. It is a direct analog to study the similar process among the quarkonia. CLEOc reported the observation of two-photon transition of $\Upsilon(3 S)$ to $\Upsilon(2 S)$ [8]. 


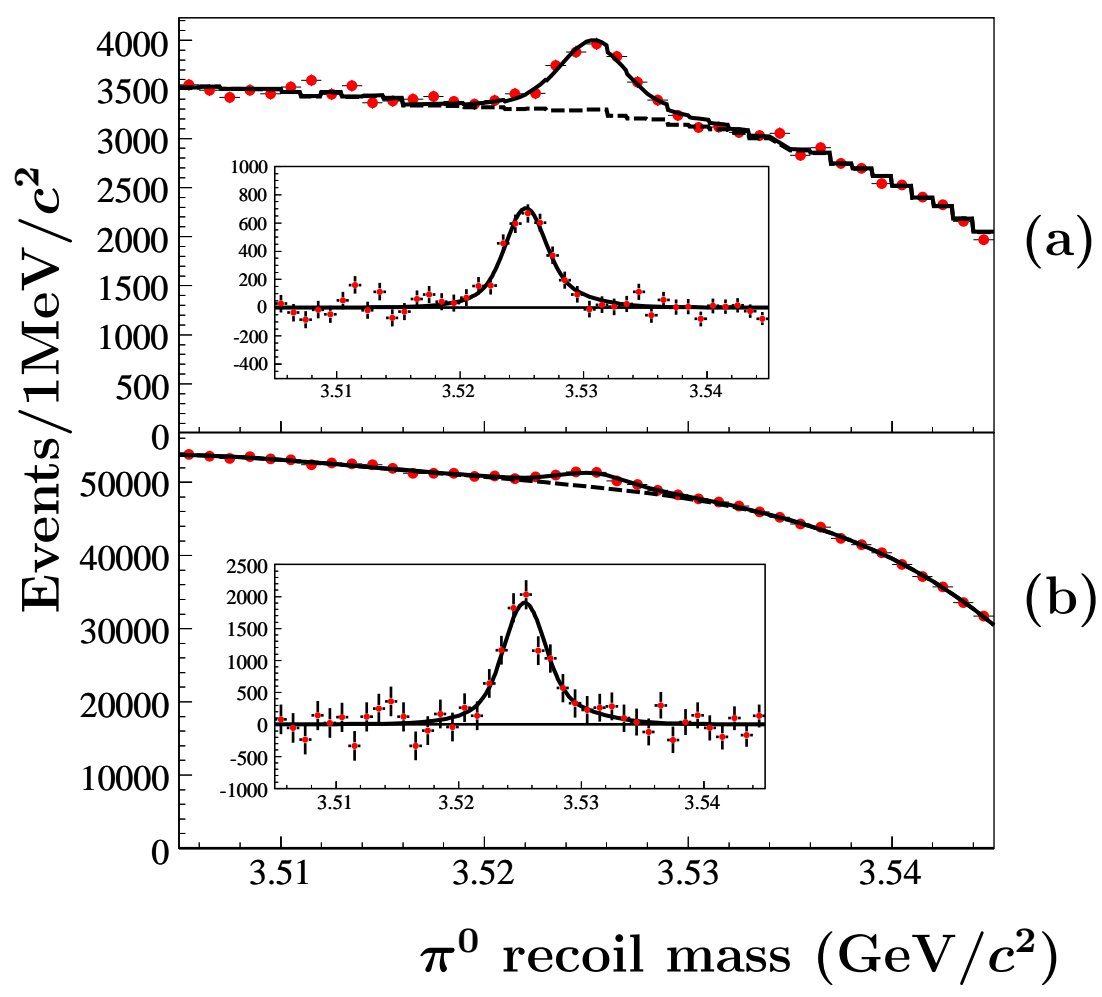

Figure 2: (a) The $\pi^{0}$ recoil mass spectrum and fit for the $E 1$-tagged analysis of $\psi^{\prime} \rightarrow \pi^{0} h_{c}, h_{c} \rightarrow \gamma \eta_{c}$; (b) the $\pi^{0}$ recoil mass spectrum and fit for the inclusive analysis of $\psi^{\prime} \rightarrow \pi^{0} h_{c}$. Fits are shown as solid lines, background as dashed lines. The insets show the background-subtracted spectra.

Charmonium is a specially interesting system because it provides a good place to test the nonperturbative Quantum ChromoDynamics(QCD) [9] . Hence, the measurement of such transitions among charmonium states will promote our understanding of QCD theory. Until now, two-photon transition in charmonium is still escaped from experimental measurement. The reason is basically due to its low branching ratio and the limited experimental data. For instance, the $J / \psi$ yield from two-photon transition from $\psi(2 S)$ is estimated to be much smaller than that through $\chi_{c J}$ intermediate states [10]. Therefore, with the broadened instrumental resolution of the spectrometer, the dominant two E1 transitions, which have same final states, make it difficult to evaluate the strength of the two-photon continuous process, if the statistics is not enough.

Taking advantage of the high luminosity of electron-positron storage ring at BEPCII and excellent spectrometer, BESIII took about 106M $\psi(2 S)$ data, 4 times of CLEOc's. This high statistics data guarantee the significance of the measurement of two-photon transition of $\psi(2 S)$ to $J / \psi$.

Here we use two decay modes to tag $J / \psi, J / \psi \rightarrow e^{+} e^{-}$and $J / \psi \rightarrow \mu^{+} \mu^{-}$. After event selection we can see some hint of the two-photon transition $\psi(2 S) \rightarrow \gamma \gamma J / \psi$ in the red boxes(signal region) on the scattering plots of $m_{\gamma L \text { recoil }}$ vs. $M_{\gamma \gamma}$, see Figure3. by studying the invariant mass distribution of the two leptons of the events in the red boxed, we can extract the yield of the signals 


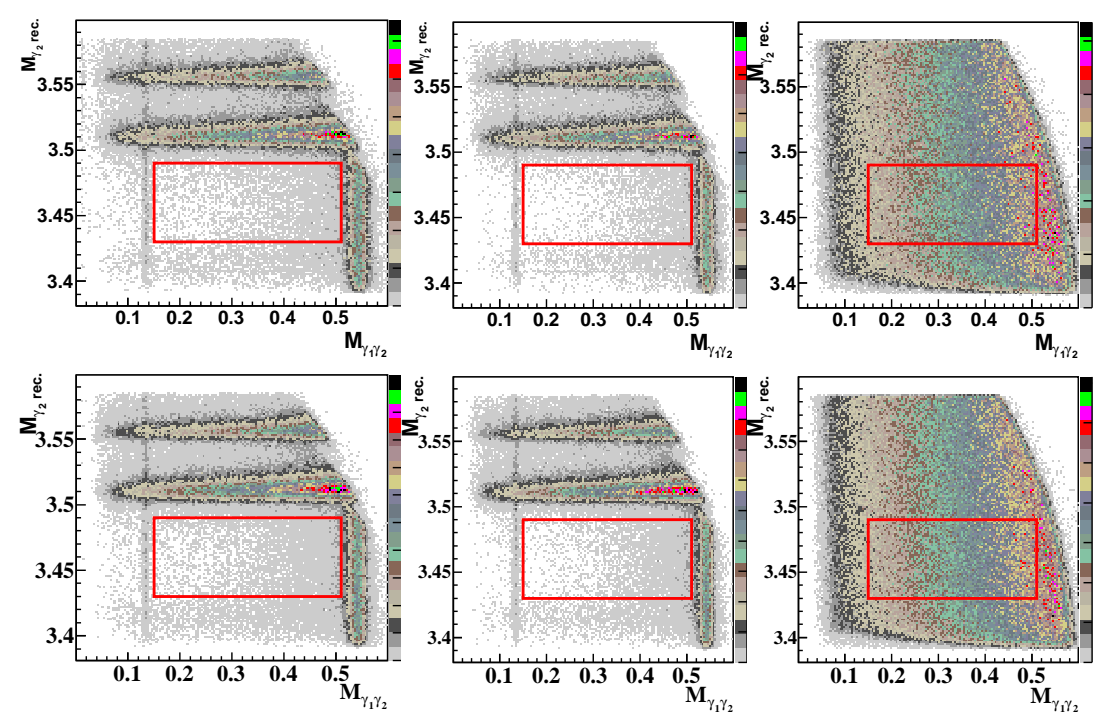

Figure 3: Scattering plots of $m_{\gamma L \text { recoil }}$ vs. $M_{\gamma \gamma}$. The upper row is $J / \psi \rightarrow e^{+} e^{-}$channels, the bottom row $J / \psi \rightarrow \mu^{+} \mu^{-}$channels; from the left to right the plots are data, inclusive Monte Carlo and phase space Monte Carlo. The events in The boxes will be used to extract the signal.

\begin{tabular}{lll}
\hline \hline & $e e$ channel & $\mu \mu$ channel \\
\hline No. of signals & $495.8 \pm 37.9$ & $615.9 \pm 40.9$ \\
acceptance & $(7.44 \pm 0.02) \%$ & $(9.92 \pm 0.02) \%$ \\
significance & $12.5 \sigma$ & $14.3 \sigma$ \\
\hline$B r\left(\psi^{\prime} \rightarrow \gamma \gamma J / \psi\right)\left[10^{-3}\right]$ & $1.06 \pm 0.08_{-0.18}^{+0.17}$ & $0.99 \pm 0.07_{-0.18}^{+0.19}$ \\
& \multicolumn{2}{c}{$1.02 \pm 0.05_{-0.20}^{+0.19}$ (average) } \\
\hline \hline
\end{tabular}

Table 1: Summary of the results of two-photon transition of $\psi(2 S) \rightarrow \gamma \gamma J / \psi$

of two-photon transition.

With the reconstruction via $e^{+} e^{-}$and $\mu^{+} \mu^{-}$channel, the plots in Figure 4 show the distribution of $m_{l^{+} l^{-}}$of the combined exclusively simulated $\psi(2 S)$ decay backgrounds, where the relative branching fractions are taken from ref. [11]. Unbinned maximum likelihood fit is performed to the selected data as shown in Figure 4, where the MC background shape and signal shape are taken from the MC simulation. The results are summarized in Table 1 , the signals of $\psi^{\prime} \rightarrow \gamma \gamma J / \psi$ twophoton transition are observed with high significances, and the result does not conflict with the up-limit assumption of $\operatorname{Br}(\psi(2 S) \rightarrow \gamma \gamma J / \psi)<1 \times 10^{3}$ by CLEO [10] .

\section{Summary}

Some recent charmonium results, obtained based on $106 \mathrm{M} \psi(2 S)$ data collected at BESIII/BEPCII, are presented. These results include the observation of $h_{c}$ from $\psi(2 s) \rightarrow \pi^{0} h_{c}$, and the observation 

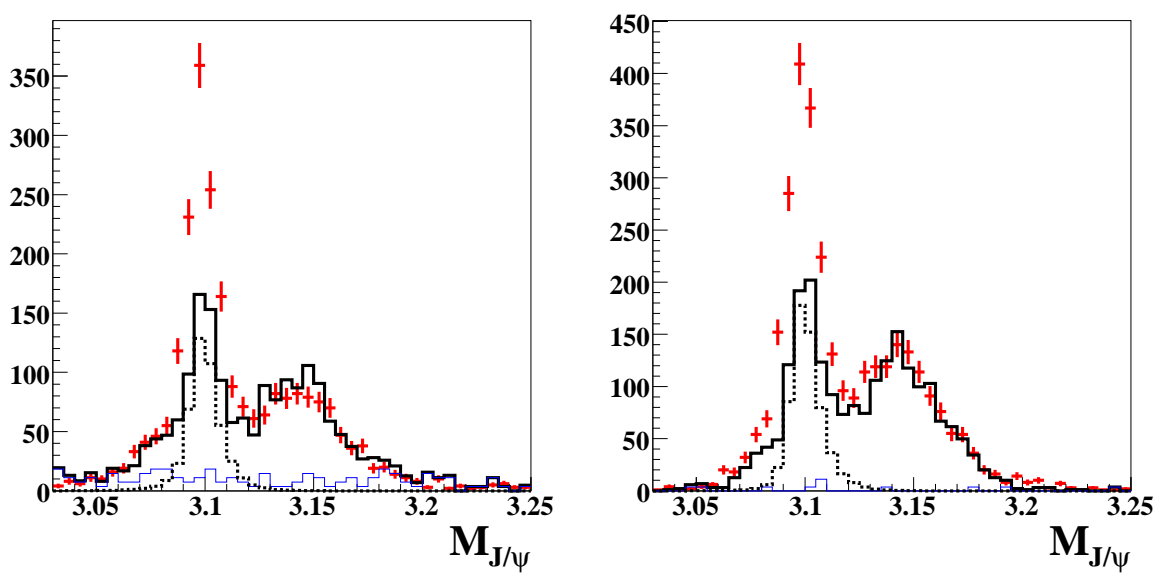

Figure 4: Scattering plots of $m_{\gamma L \text { recoil }}$ vs. $M_{\gamma \gamma}$. The upper row is $J / \psi \rightarrow e^{+} e^{-}$channels, the bottom row $J / \psi \rightarrow \mu^{+} \mu^{-}$channels; from the left to right the plots are data, inclusive Monte Carlo and phase space Monte Carlo. The events in The boxes will be used to extract the signal.

of two-photon transition of $\psi(2 S) \rightarrow \gamma \gamma J / \psi$. Some of them are observed/measured for the first time in the world, which include the measurements of $\operatorname{Br}\left(\psi(2 S) \rightarrow \gamma h_{c}\right.$ and $B r\left(h_{c} \rightarrow \gamma h_{c}\right)$ separately, the width of $h_{c}$, and that of $\operatorname{Br}(\psi(2 S) \rightarrow \gamma \gamma J / \psi)$. More exciting results at BES-III, which will improve our knowledge at this energy region, are coming soon.

\section{References}

[1] M. Ablikim et al. The construction of the BESIII experiment, Nucl. Instrum. Meth. A 598, 7 (2009).

[2] S. Dobbs et al., Precision Measurement of the Mass of the $h_{c}\left({ }^{1} P_{1}\right)$ State of Charmonium Phys. Rev. Lett. 101, 182003 (2008) [hep-ex/ 0805 . 4599].

[3] M. Ablikim et al., Measurements of $h_{c}\left({ }^{1} P_{1}\right)$ in $\psi^{\prime}$ Decays, Phys. Rev. Lett. 104, 132002 (2010) [hep-ex/1002.0501].

[4] A. Quattropani, F. Bassani and S. Carillo, Two-photon transitions to excited states in atomic hydrogen, Phys. Rev. A 25, 3079-3089 (1982)

[5] F. Bassani, J. J. Forney and A. Quattropani, Choice of gauge in two-photon transitions: $1 S-2 S$ transition in atomic hydrogen, Phys. Rev. Lett. 39, 1070-1073 (1977).

[6] A. Quattropani, and F. Bassani, Two Photon Transitions In Positronium, Phys. Rev. Lett. 50, 1258-1260 (1983).

[7] E. E. Kholupenko, and A. V. Ivanchik, Two-Photon $2 S-1 S$ Transitions during Recombination of Hydrogen in the Universe, Astron. Lett. 32, 795-803 (2006). [astro-ph/ 0611395].

[8] F. Butler, et al., Analysis of hadronic transitions in $\Upsilon(3 S)$ decays, Phys. Rev. D49, 40-57 (1994).

[9] D. M. Asner, et al., Physics at BES-III, International Journal of Modern Physics A Volume: 24, Issue: 1 supp (2009).

[10] H. Mendez, et al., Branching Fractions for Transitions of $\psi(2 S)$ to J/ $\psi$, Phys. Rev. D78, 011102 (2008). 
[11] K. Nakamura et al., The Review of Particle Physics, J. Phys. G 37, 075021 (2010) 